\title{
La evaluación externa: tensiones entre la educación pública y la educación privada
}

\author{
Marta OSORIO DE SARMIENTO* y Julio Esteban UNIVIO MOLANO**
}

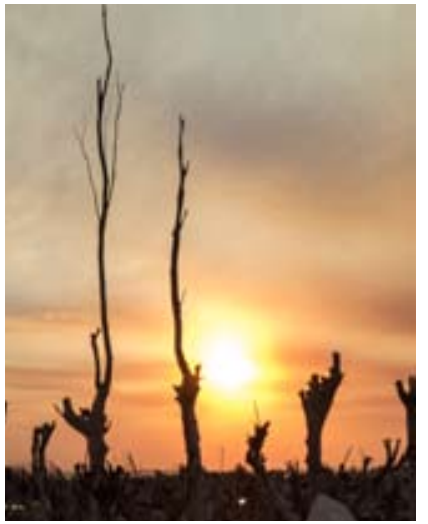

Detalle $\mathbf{S} / \mathbf{T}$, fotografía. Patricia Bonjour Universidad de Granada España; MBA en gestión y liderazgo por el Instituto Europeo de Posgrados; Especialista en dirección de centros educativos por la Universidad de la Sabana, Decana de la Escuela de Educación y Ciencias Sociales de la Fundación Universitaria Juan N. Corpas. Colombia | marta.osorio@juanncorpas.edu.co

** Graduado en Filosofía en la Universidad Nacional de Colombia, candidato a doctor en educación por la Universidad de Granada, España. Docente investigador de la Universidad Libre de Colombia, Bogotá. Colombia | univioesteban@gmail.com
Resumen

Este artículo es el resultado de una investigación dirigida a analizar los resultados alcanzados por los estudiantes de las instituciones públicas y privadas de secundaria, en la aplicación de la prueba externa conocida como Saber 11, durante 2011, 2012 y 2013. El propósito ha sido determinar cómo, a partir de estos resultados, se ha establecido, por parte del Ministerio de Educación Nacional y los medios de comunicación, una marcada discriminación entre la educación pública y la privada, en términos de la calidad educativa.

Palabras clave: evaluación externa; calidad de la educación; rendimiento académico

\begin{abstract}
External Evaluation: Tensions between the Public and Private Education
\end{abstract}

\begin{abstract}
This article is the result of a research aimed to analyze the results achieved by students of public and private secondary schools in the application of the external test known as Saber 11 during 2011, 2012 and 2013, in order to determine how, on the basis of these results, the Ministry of National Education and the media has established a marked discrimination between public and private education in terms of educational quality
\end{abstract}

Key words: external evaluation; quality of education; academic performance

\section{Incidencia de las pruebas externas en la calidad de la educación media}

$\mathrm{E}$ n Colombia, a partir de la década de los ochenta del siglo pasado, se da inicio a un proceso de reformas en la educación, centrado preferencialmente en el desempeño de los estudiantes. Por tal razón, en 1982, el Ministerio de Educación Nacional (MEN), a través de la División de Evaluación de Rendimiento Escolar, propuso mecanismos para determinar el alcance de los logros de los estudiantes de la educación básica y media en el marco de la renovación curricular. La División de Evaluación, ante la falta de continuidad detectada en los procesos evaluativos, diseñó una evaluación basada en el enfoque de sistemas y la evaluación de procesos. Conforme con esto, se determinó que los objetivos de la evaluación estuvieran relacionados con la filosofía y el enfoque de la renovación, los planes y proyectos de la administración escolar, el ambiente educati- 
vo, físico y sociocultural, la capacitación de los docentes, el desempeño de los estudiantes, la relación con los padres de familia, el seguimiento y asesoría de las instituciones.

De acuerdo con la intencionalidad de mejorar la calidad educativa, en la década de los noventa y en la primera del presente siglo, las estrategias gubernamentales propuestas a través del MEN, tienden claramente a fijar su atención en las pruebas externas que se aplican a los estudiantes de los niveles básico y medio de escolaridad, mediante la expedición de normas que pretenden tanto su reglamentación como la transformación de las mismas. En este sentido, las pruebas Saber $11^{1}$ aplicadas a los estudiantes de último grado de la educación media, se van transformando en su contenido y por ende en su enfoque, pues además de establecerlas como requisito para ingreso a la educación superior, ellas suministran información acerca de los procesos de enseñanza y de aprendizaje. Los resultados se emplean para rankear y comparar la educación media pública con la educación media privada, clasificando a los colegios mediante categorías como muy superior, superior, alto, medio, bajo, inferior y muy inferior.

Se puede afirmar que a pesar de las políticas educativas y las diversas estrategias enfocadas a mejorar la calidad de la educación, los resultados obtenidos en las pruebas externas indican escasos avances en la superación de las deficiencias en los aprendizajes de los estudiantes. Esto lleva a interrogarse sobre la incidencia que pueda tener este tipo de evaluación en el mejoramiento de la calidad educativa y sobre si ésta es un criterio adecuado para establecer una auténtica comparación entre la educación pública con la privada, en el marco del desafío que implica para el país el mejoramiento y aseguramiento de la calidad de los procesos de enseñanza y de aprendizaje, así como de sus métodos y didácticas. Para dar respuesta a estos interrogantes es pertinente realizar diferencias entre los tipos de evaluación que se orientan a dichos objetivos.

\section{Evaluación interna}

En las instituciones educativas se han aplicado distintas formas de mantener la calidad de la educación a través de la evaluación, entre las que se destaca la que realiza el docente durante el trabajo pedagógico en el aula. Ésta comúnmente se conoce como interna y le permite al educador hacer un seguimiento sobre el proceso de aprendizaje de sus estudiantes y buscar mecanismos para mejorar sus deficiencias. Este tipo de evaluación suscita múltiples preocupaciones y reflexiones. Un ejemplo es lo expresado por la directora del ICFES, Ximena Dueñas Herrera, en el marco del V Seminario Internacional de Investigación sobre la Calidad de la Educación, al presentar los hallazgos de la entidad sobre los factores que inciden en los resultados académicos de los estudiantes y que fue recogida en una entrevista ofrecida por ella a la Revista Semana.com:

En el país hace falta hacer investigación educativa. (...). Entonces, ¿qué hace que un estudiante tenga buenos resultados académicos? Los investigadores coinciden en que primero hay que llevar esta pregunta más atrás. ¿Qué significa tener buenos resultados? ¿Un buen puntaje en pruebas estandarizadas o una buena nota en la materia? ¿Es mejor un estudiante que mejora su desempeño o uno que mantiene excelentes calificaciones? Todo, dicen los expertos, depende del contexto y de los indicadores que cada país o institución determine: la calidad de la educación se construye con múltiples factores (2014).

Este planteamiento sustenta la necesidad de investigar rigurosamente los factores que inciden en el desempeño de los estudiantes: el método de estudio, el tiempo dedicado a las tareas, el ambiente escolar, los compañeros, la discriminación, la influencia de los medios de comunicación, y los asuntos familiares, económicos y sociales. Necesidad que ha reconocido el ICFES en palabras de Carolina Lopera, directora de la Oficina de Investigaciones de esta entidad, quien a partir de un estudio sobre el contexto escolar y social del aprendizaje en Colombia, afirmó lo siguiente en el V Seminario Internacional de Investigación sobre Calidad de la Educación:

El estudio encontró que el nivel socioeconómico de los estudiantes explica por lo menos el 20 por ciento de la varianza en el puntaje. Aparte de esto hay unos factores en el hogar que nos ayudaron a entender cómo el con- 
texto incide en los resultados académicos de los estudiantes (...). Encontramos que hay estilos parentales permisivos y negligentes que hacen que los estudiantes tengan hasta 20 puntos menos en el logro educativo. (...) Cerca del 50 por ciento de la población tiene papás asertivos, afortunadamente, mientras que 2 de cada 10 tienen padres negligentes o permisivos y eso no cambia mucho ni por nivel socioeconómico ni por zonas rurales o urbanas. (semana.com, 2014)

Dicho estudio y las posiciones expresadas por Lopera hacen evidente que se deben reconocer los factores que intervienen en los desempeños académicos de los estudiantes y que éstos requieren ser visibilizados y relacionados con los resultados de las pruebas externas que se les aplican; sin desconocer los factores asociados con el desempeño de los docentes como la vocación, la formación profesional, la capacitación que reciben, el conocimiento de métodos y estrategias pedagógicas, la comunicación y el amor por la enseñanza, entre otros que, sin duda, marcan la motivación del estudiante por el aprendizaje.

\section{Evaluación externa}

En Colombia, el Instituto Colombiano para el Fomento de la Educación Superior(ICFES), es la entidad encargada de aplicar las pruebas Saber 11 y otras evaluaciones estandarizadas a los estudiantes de las instituciones educativas públicas y privadas. De acuerdo con la Secretaría de Educación del Distrito de Bogotá (SED):

La evaluación externa está compuesta por las pruebas objetivas distritales, nacionales $e$ internacionales, centradas en parámetros estandarizados y aplicadas por entidades diferentes al colegio. Estas evaluaciones y estudios brindan información importante a los colegios y a las autoridades educativas, y aunque la SED no las considera como el único indicador de calidad educativa, sus resultados pueden revelar tendencias que deben ser objeto de análisis y deben ser tenidos en cuenta porque orientan la labor educativa hacia la calidad esperada y son un importante referente en la construcción del POA [Plan Operativo Anual] y en los planes de mejoramiento (SED, s.f).
Las evaluaciones externas o estandarizadas, como comunmente se les conoce, le permiten al ICFES obtener informes de los resultados obtenidos por los estudiantes de las distintas instituciones educativas, tanto públicas como privadas, con el fin de establecer la clasificación de las mismas en términos de calidad educativa.

Se debe tener en cuenta que lo realizado con la evaluación externa, no necesariamente significa desconocer lo que se ha ejecutado con la evaluación interna, es decir, que con esta última se pretende que, una vez corregidas las deficiencias en el proceso enseñanza aprendizaje, la primera resulte satisfactoria o favorable para las instituciones, tanto públicas como privadas, pues su finalidad no es otra que la de servir de indicador de la calidad educativa de la institución.

La prueba externa Saber 11 es un instrumento de evaluación estandarizado que tiene como propósito fundamental la medición del nivel de desarrollo de las competencias básicas de los estudiantes que están por terminar su ciclo de formación en la educación media. Los resultados de esta prueba son tomados, por parte del ICFES y del MEN, como un indicador de calidad de la educación y, por lo tanto, del rendimiento académico de los estudiantes. Esta prueba está constituida estructuralmente por dos componentes denominados núcleo común y componente flexible:

En el primero, están las áreas que se consideran fundamentales, de acuerdo con la Ley General de Educación y los estándares básicos de competencias. Estas son: lenguaje, matemáticas, biología, química, física, filosofía, ciencias sociales e inglés. Todos los estudiantes deben presentar las pruebas que conforman este núcleo. El componente flexible está integrado por dos clases de pruebas: de profundización e interdisciplinares. Su propósito es evaluar áreas de interés para los estudiantes, quienes pueden escoger aquella que más se ajuste a sus intereses y dominio académico (ICFES,2010, p. 10).

Existe, sin embargo, un grado de desconfianza por parte del cuerpo docente del sector público, pues con la implementación de este tipo de pruebas se desconoce el contexto social en el que se encuentra la institución educativa. 
Además, porque no se sabe cuáles consecuencias puede haber para las instituciones cuyos resultados en las pruebas sean deficientes $y$, particularmente, para los docentes. Por otro lado, tampoco se les pregunta a las instituciones, tanto públicas como privadas, si están o no de acuerdo con los resultados obtenidos para emprender las respectivas acciones de mejoramiento.

\section{Calidad de la educación}

Actualmente, la preocupación por la calidad de la educación por parte del gobierno en Colombia está estrechamente relacionada con las políticas económicas internacionales, con organizaciones internacionales dedicadas a la cooperación y financiación de la educación. Estas organizaciones participan en las apreciaciones sobre la calidad del sistema educativo nacional de la educación media, a través de evaluaciones externas como las evaluaciones Pisa. ${ }^{2}$

En el ámbito nacional, el Estado se responsabiliza de la gestión por la promoción de la calidad educativa. Ésta es la razón de ser de un ente como el ICFES, institución dedicada a establecer el sistema nacional de la medición de la calidad de la educación. Su propósito es instaurar un sistema de control a través del cual obtener información sobre los estándares de calidad de la educación y divulgarla, mediante la aplicación de pruebas externas, por ejemplo, la prueba Saber 11.

La expresión "calidad de la educación", es una de las más empleadas en el campo educativo para referirse al desempeño académico que tienen los estudiantes de los distintos niveles escolares y se relaciona, básicamente, con el mejoramiento educativo, la eficacia y la eficiencia, vinculadas con la medición de logros del aprendizaje, a través de las evaluaciones externas. Sin embargo, la significación del concepto es dinámica, dado que su naturaleza obedece, también, a los cambios del sistema educativo, influenciados por las transformaciones sociales, políticas, culturales, educativas y tecnológicas del mundo. De acuerdo con el MEN:

Una educación de calidad es aquella que forma mejores seres humanos, ciudadanos con valores éticos, respetuosos de lo público, que ejercen los derechos humanos, cumplen con sus deberes y conviven en paz. Una educación que genera oportunidades legítimas de

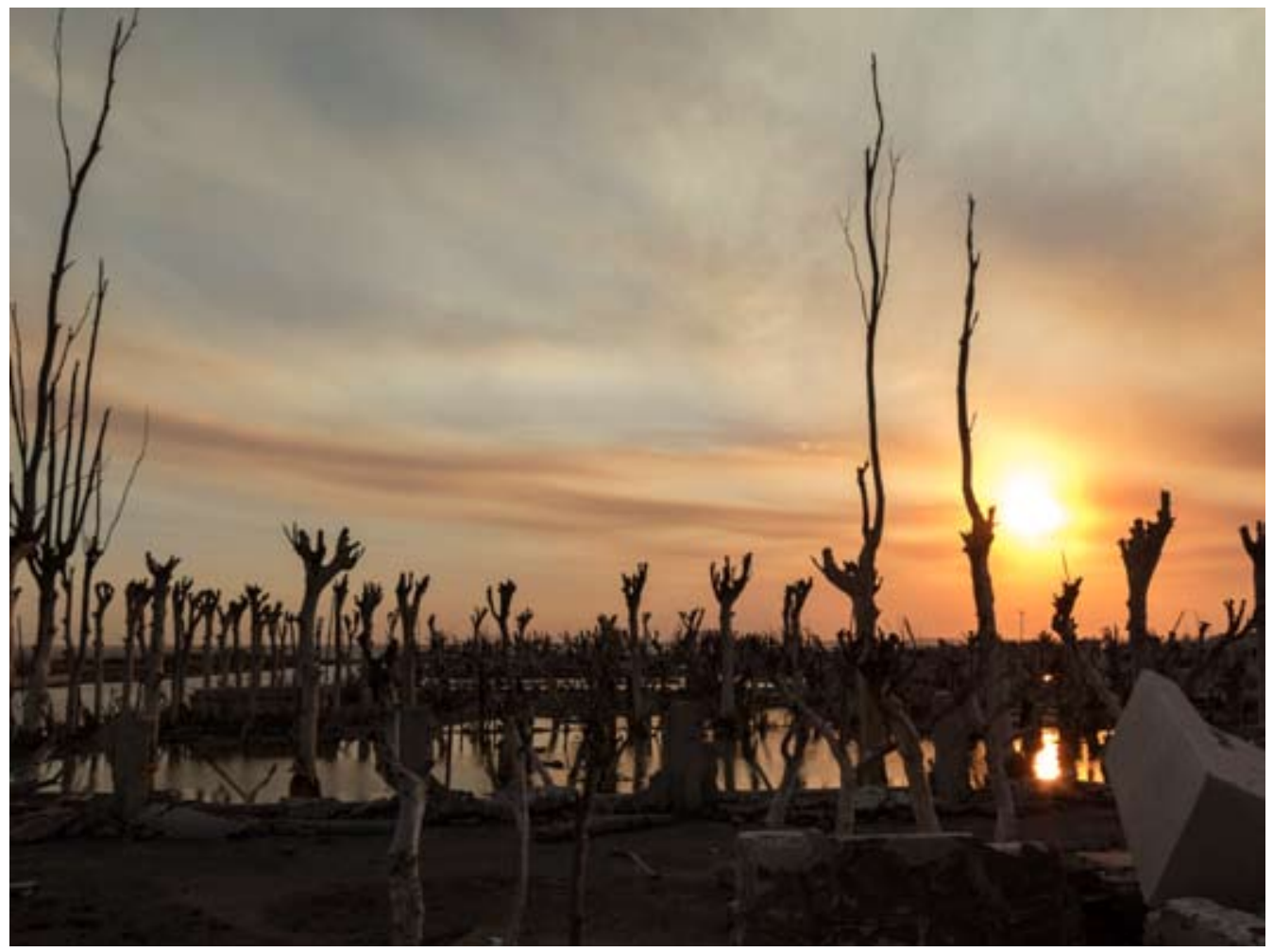

S/T, fotografía. Patricia Bonjour 
progreso y prosperidad para ellos y para el país (2010: 4-5).

La presión que ejercen los cambios políticos y económicos de orden mundial en las reformas a la educación, para adecuarla a los nuevos criterios de producción, ha llevado a algunos expertos, como Magendzo, a afirmar que

"las analogías con las cuales la educación es comparada, bajo la óptica racional, provee de una serie de metáforas que ligan la calidad de la educación con la empresa, al alumno con el cliente, a la mente con la máquina, a las ideas con productos, al currículo con los artículos de consumo..." (Magendzo citado en Mejía, 2000: 1).

Posturas como ésta revelan las controversias existentes sobre el término; pese a las cuales es necesario reconocer que la medición de la calidad de la educación media, con base en la evaluación externa, es un aspecto fundamental para los procesos de mejoramiento educativo.

\section{Rendimiento académico}

En el desarrollo de los procesos de aprendizaje, se ponen en juego las distintas capacidades de los estudiantes, respecto a su relación con su formación intelectual, social, científica, política, ética y espiritual, entre otras. De acuerdo con los diversos estudios realizados sobre los procesos de aprendizaje, estas capacidades son las que evidencian el rendimiento académico de los estudiantes. El análisis de este factor se centra en dos aspectos fundamentales: el proceso y los resultados, mediante los cuales es posible realizar interpretaciones de orden cuantitativo o cualitativo.

En este sentido, las Pruebas Saber 11 están pensadas como un mecanismo de medición de la calidad educativa y también del rendimiento académico (Gómez, 2010), por lo que les sirven a los directivos de las instituciones educativas, tanto públicas como privadas, para identificar en qué estado de desarrollo de competencias y alcance de logros se encuentran sus estudiantes y tener como referente el mejoramiento educativo. Con respecto a los docentes, el conocimiento de los resultados obtenidos por sus estudiantes en estas pruebas les permite hacer una autorreflexión acerca del desarrollo de las competencias de sus alumnos/as y buscar los mecanismos más adecuados para alcanzar en un futuro mejores resultados. Igualmente, se constituyen en una base para el gobierno y las entidades estatales que participan en la regulación de la educación para trazar políticas educativas y diseñar mecanismos de acompañamiento a las instituciones.

\section{Metodología}

El estudio realizado se clasifica como una investigación mixta en cuyo análisis cualitativo se examinaron documentos oficiales sobre la evaluación, y en el cuantitativo, resultados de las pruebas Saber 11, empleando el software SPSS ${ }^{3}$ como complemento para la interpretación de los mismos.

Con base en un enfoque descriptivo-interpretativo, se analizaron los resultados de la evaluación Saber 11, en 12.273 instituciones educativas, entre oficiales y privadas de la educación media en el país, que es el $100 \%$. De éstos, se tomaron los cien (100) mejores colegios incluyendo los de la ciudad de Bogotá D.C., entre 2011 y 2013, con base en las frecuencias absolutas de cada observación, que en este caso corresponden a estos cien (100) colegios, destacándose el mínimo, la mediana y el máximo de cada año. Además, se muestra en el análisis del cociente de cada frecuencia con respecto a la suma total de las observaciones (colegios), es decir, la frecuencia relativa (Canavos, 1998) que, en este caso, es el porcentaje (\%) de participación de los cien (100) de acuerdo con su naturaleza en el período segmentado.

Para dicho análisis, se tuvieron en cuenta los resultados de las pruebas Saber 11 aplicadas por el ICFES entre 2011 y 2013, de acuerdo con las jornadas de la mañana, la tarde, la noche y la completa u ordinaria ${ }^{4}$, así también el carácter público y privado de las instituciones.

\section{Resultados}

El análisis de los datos y la información permitió observar que, de los doce mil doscientos setenta y tres (12.273) registros anuales (esto debido a que una sola institución tiene más de una jornada) de los colegios que presentaron la Prueba ICFES Saber, 11,38 \% son de la jornada de la mañana, $31 \%$ de la jornada 
completa u ordinaria, $13 \%$ de la jornada de la tarde y $12 \%$ de la jornada de la noche. El análisis tuvo en cuenta el promedio de participación, expresado en porcentajes entre 2011 y 2013, de los colegios según su naturaleza (oficial o no oficial).

En el caso de la asignatura de matemáticas en las jornadas de la mañana y de la tarde, la participación en los colegios de naturaleza no oficial es del $89 \%$ con respecto al $11 \%$ de los colegios de naturaleza oficial (ver Tabla Nro. 1 ), en contraste con la jornada completa u ordinaria que es del $99 \%$ de naturaleza oficial (ver Tabla Nro. 2).

La Tabla Nro. 3 expone los consolidados promedios de los puntajes de matemáticas por jornadas y años.

En el caso de la asignatura química, el promedio de participación de los colegios de naturaleza oficial entre los cien (100) mejores, en el periodo de observación fue mayor: $67 \%$ en la jornada tarde y $52 \%$ en la jornada noche. Cabe anotar que son muy pocos los colegios no oficiales que ofrecen estas jornadas.

Para el caso del área de filosofía, en promedio, el $90 \%$ de los colegios privados tuvieron los mejores puntajes en jornada de la mañana, mientras que los colegios de la jornada de la tarde tuvieron el $32 \%$ de participación promedio. A pesar de que no son muchos los colegios de naturaleza privada que tienen esta jornada, comparados con los colegios públicos o de naturaleza oficial, los resultados hallados se representan en las Tablas Nro. 10, 11, 12 y 13í.

Para hacer más explícitos los resultados en el área de filosofía, se hace un comparativo entre jornadas: mañana, tarde, noche, completa ordinaria, del año 2011 al 2013, evidenciando los puntajes, tal como se indica en la Tabla Nro.8.

Tabla No 1. Promedios de matemáticas en las jornadas de la mañana y de la tarde.

\begin{tabular}{lcccccc}
\multicolumn{1}{c}{ JORNADA } & \multicolumn{3}{c}{ MAÑANA } & \multicolumn{3}{c}{ TARDE } \\
\hline Promedio puntaje en años & $\mathbf{2 0 1 1}$ & $\mathbf{2 0 1 2}$ & $\mathbf{2 0 1 3}$ & $\mathbf{2 0 1 1}$ & $\mathbf{2 0 1 2}$ & $\mathbf{2 0 1 3}$ \\
\hline Puntaje máximo & 83.53 & 80.68 & 78.3 & 64.18 & 63.76 & 82.23 \\
Mediana puntaje (50 \%) & 63.6 & 62.1 & 63.11 & 53.21 & 52.69 & 51.33 \\
Puntaje mínimo & 59.44 & 59.2 & 59.18 & 51.40 & 50.27 & 48.9 \\
\hline
\end{tabular}

Fuente: elaboración propia con base en datos del ICFES.

Tabla No 2. Promedios de matemáticas en las jornadas completa u ordinaria y de la noche.

\begin{tabular}{lcccccc}
\multicolumn{1}{c}{ JORNADA } & \multicolumn{2}{c}{ COMPLETA U ORDINARIA } & \multicolumn{3}{c}{ NOCHE } \\
\hline Promedio puntaje en años & $\mathbf{2 0 1 1}$ & $\mathbf{2 0 1 2}$ & $\mathbf{2 0 1 3}$ & $\mathbf{2 0 1 1}$ & $\mathbf{2 0 1 2}$ & $\mathbf{2 0 1 3}$ \\
\hline Puntaje máximo & 96.59 & 79.33 & 92.21 & 69 & 56.88 & 58.5 \\
Mediana puntaje (50 \%) & 68.67 & 69.78 & 70.61 & 46.95 & 45.67 & 44.64 \\
Puntaje mínimo & 65.59 & 60.58 & 66.67 & 44.85 & 44.68 & 43.46 \\
\hline
\end{tabular}

Fuente: elaboración propia con base en datos del ICFES.

Tabla No 3. Consolidados de matemáticas.

\begin{tabular}{lcccccccccccc}
\multicolumn{1}{c}{ JORNADA } & \multicolumn{3}{c}{ MAÑANA } & & TARDE & & \multicolumn{2}{c}{ COMPLETA U ORDINARIA } & NOCHE \\
\hline $\begin{array}{l}\text { Promedio puntaje } \\
\text { en años }\end{array}$ & $\mathbf{2 0 1 1}$ & $\mathbf{2 0 1 2}$ & $\mathbf{2 0 1 3}$ & $\mathbf{2 0 1 1}$ & $\mathbf{2 0 1 2}$ & $\mathbf{2 0 1 3}$ & $\mathbf{2 0 1 1}$ & $\mathbf{2 0 1 2}$ & $\mathbf{2 0 1 3}$ & $\mathbf{2 0 1 1}$ & $\mathbf{2 0 1 2}$ & $\mathbf{2 0 1 3}$ \\
\hline Mediana puntaje (50\%) & 83.53 & 80.68 & 78.3 & 64.18 & 63.76 & 82.23 & 96.59 & 79.33 & 92.21 & 69 & 56.88 & 58.5 \\
Puntaje mínimo & 63.6 & 62.1 & 63.11 & 53.21 & 52.69 & 51.33 & 68.67 & 69.78 & 70.61 & 46.95 & 45.67 & 44.64 \\
Puntaje máximo & 59.44 & 59.2 & 59.18 & 51.40 & 50.27 & 48.9 & 65.59 & 60.58 & 66.67 & 44.85 & 44.68 & 43.46 \\
\hline
\end{tabular}

Fuente: elaboración propia con base en datos del ICFES.

Tabla No 4. Promedios de química por años y jornadas.

\begin{tabular}{lcccccc}
\multicolumn{1}{c}{ JORNADA } & \multicolumn{3}{c}{ MAÑANA } & \multicolumn{3}{c}{ TARDE } \\
\hline Naturaleza/Años & $\mathbf{2 0 1 1}$ & $\mathbf{2 0 1 2}$ & $\mathbf{2 0 1 3}$ & $\mathbf{2 0 1 1}$ & $\mathbf{2 0 1 2}$ & $\mathbf{2 0 1 3}$ \\
\hline No oficial & $91 \%$ & $91 \%$ & $91 \%$ & $31 \%$ & $35 \%$ & $37 \%$ \\
Oficial & $9 \%$ & $9 \%$ & $9 \%$ & $69 \%$ & $65 \%$ & $63 \%$ \\
\hline
\end{tabular}


Tabla No 5. Porcentajes por jornada en química en la mañana y en la tarde.

\section{NATURALEZA/JORNADA}

No oficial

Oficial
MAÑANA

$99 \%$

$1 \%$
TARDE

$33 \%$

$67 \%$

Fuente: elaboración propia con base en datos del ICFES.

Tabla No 6. Promedios de filosofía por años y jornadas de la mañana y la tarde.

\begin{tabular}{lcccccc}
\multicolumn{1}{c}{ JORNADA } & \multicolumn{3}{c}{ MAÑANA } & \multicolumn{3}{c}{ TARDE } \\
\hline Promedio puntaje en años & $\mathbf{2 0 1 1}$ & $\mathbf{2 0 1 2}$ & $\mathbf{2 0 1 3}$ & $\mathbf{2 0 1 1}$ & $\mathbf{2 0 1 2}$ & $\mathbf{2 0 1 3}$ \\
\hline No oficial & $88 \%$ & $89 \%$ & $93 \%$ & $28 \%$ & $28 \%$ & $39 \%$ \\
Oficial & $12 \%$ & $11 \%$ & $7 \%$ & $72 \%$ & $72 \%$ & $61 \%$ \\
\hline
\end{tabular}

Fuente: elaboración propia con base en datos del ICFES.

Tabla No 7. Porcentajes de filosofía por años y jornadas de completa u ordinaria y la noche

\begin{tabular}{lcccccc}
\multicolumn{1}{c}{ JORNADA/NATURALEZA } & NOCHE & \multicolumn{3}{c}{ COMPLETA U ORDINARIA } \\
\hline Años & $\mathbf{2 0 1 1}$ & $\mathbf{2 0 1 2}$ & $\mathbf{2 0 1 3}$ & $\mathbf{2 0 1 1}$ & $\mathbf{2 0 1 2}$ & $\mathbf{2 0 1 3}$ \\
\hline No oficial & $67 \%$ & $56 \%$ & $55 \%$ & $98 \%$ & $100 \%$ & $97 \%$ \\
Oficial & $33 \%$ & $44 \%$ & $45 \%$ & $2 \%$ & $0 \%$ & $3 \%$ \\
\hline
\end{tabular}

Fuente: elaboración propia con base en datos del ICFES.

Tabla No 8. Consolidados de filosofía.

\begin{tabular}{lcccccccccccc}
\multicolumn{1}{c}{ JORNADA } & \multicolumn{3}{c}{ MAÑANA } & & TARDE & & & NOCHE & & COMPLETA U ORDINARIA \\
\hline $\begin{array}{l}\text { Promedio puntaje } \\
\text { en años }\end{array}$ & $\mathbf{2 0 1 1}$ & $\mathbf{2 0 1 2}$ & $\mathbf{2 0 1 3}$ & $\mathbf{2 0 1 1}$ & $\mathbf{2 0 1 2}$ & $\mathbf{2 0 1 3}$ & $\mathbf{2 0 1 1}$ & $\mathbf{2 0 1 2}$ & $\mathbf{2 0 1 3}$ & $\mathbf{2 0 1 1}$ & $\mathbf{2 0 1 2}$ & $\mathbf{2 0 1 3}$ \\
\hline Mediana puntaje (50\%) & 52.09 & 50.66 & 52.94 & 47.16 & 45.18 & 45.55 & 43.08 & 41.15 & 40.71 & 54.48 & 56.78 & 57.58 \\
Puntaje mínimo & 50.69 & 48.7 & 50 & 45.57 & 44.25 & 43.78 & 41.2 & 40.13 & 39.36 & 52.91 & 53.6 & 55.11 \\
Puntaje máximo & 60.00 & 62.09 & 64.26 & 58.68 & 51.17 & 59.04 & 59.39 & 55 & 50.5 & 65 & 65.12 & 65.44 \\
\hline
\end{tabular}

Fuente: elaboración propia con base en datos del ICFES.

Tabla No 9. Porcentajes por jornada en lenguaje en la mañana y en la tarde.

\begin{tabular}{lcc}
\multicolumn{1}{c}{ NATURALEZA/JORNADA } & MAÑANA & TARDE \\
\hline No oficial & $95 \%$ & $39 \%$ \\
Oficial & $5 \%$ & $61 \%$ \\
\hline
\end{tabular}

Fuente: elaboración propia con base en datos del ICFES.

Tabla No 10. Porcentajes por jornada en lenguaje en la noche y completa $u$ ordinaria.

\begin{tabular}{lcc}
\multicolumn{1}{c}{ NATURALEZA/JORNADA } & NOCHE & COMPLETA U ORDINARIA \\
\hline No oficial & $75 \%$ & $98 \%$ \\
Oficial & $25 \%$ & $2 \%$ \\
\hline
\end{tabular}

Fuente: elaboración propia con base en datos del ICFES.

Tabla No 11. Consolidados de lenguaje.

\begin{tabular}{|c|c|c|c|c|c|c|c|c|c|c|c|c|}
\hline \multirow{2}{*}{$\begin{array}{c}\text { JORNADA } \\
\begin{array}{c}\text { Promedio puntaje } \\
\text { en años }\end{array} \\
\end{array}$} & \multicolumn{3}{|c|}{ MAÑANA } & \multicolumn{3}{|c|}{ TARDE } & \multicolumn{3}{|c|}{ NOCHE } & \multicolumn{3}{|c|}{ COMPLETA U ORDINARIA } \\
\hline & 2011 & 2012 & 2013 & 2011 & 2012 & 2013 & 2011 & 2012 & 2013 & 2011 & 2012 & 2013 \\
\hline Puntaje mínimo & 57.35 & 55.37 & 56 & 51.19 & 49.8 & 50.6 & 48.02 & 48 & 47.08 & 59.65 & 59.77 & 60.16 \\
\hline Puntaje máximo & 65.69 & 63.8 & 67.64 & 65.12 & 56.39 & 59.15 & 60 & 61 & 57.5 & 74.29 & 67.44 & 68.81 \\
\hline
\end{tabular}

Fuente: elaboración propia con base en datos del ICFES. 
Para el caso de lenguaje, los colegios oficiales en la jornada de la mañana sólo tuvieron en promedio un $5 \%$ de participación.

La Tabla Nro. 11 muestra el promedio del puntaje obtenido en lenguaje desde 2011 a 2013 en todas las jornadas.

Este análisis refleja, de manera global, una situación crítica de los colegios oficiales en los resultados y coincide con las diversas posiciones que señalan un bajo nivel académico con relación al de las instituciones no oficiales (privadas), evidenciando de manera clara la existencia de la brecha de la calidad educativa entre el sector educativo oficial y sector educativo privado, como resultado de los puntajes obtenidos en las cuatro asignaturas analizadas.

Además, se debe tener en cuenta que, de acuerdo con los resultados nacionales obtenidos, el comparativo entre 2011, 2012 y 2013, evidenciados en las tablas y sus porcentajes anteriores en las cuatro áreas seleccionadas (matemáticas, química, filosofía, y lenguaje), el porcentaje más alto en los tres años consecutivos lo obtuvo la jornada completa u ordinaria, no oficial, seguida de la jornada de la mañana, no oficial. En el tercer lugar, se ubicaron las jornadas: noche no oficial, tarde oficial, y noche no oficial, y en cuarto lugar las jornadas tarde oficial, noche oficial, noche no oficial y tarde oficial. Este hecho coincide con los hallazgos de Bonilla (2011) quien indica que los estudiantes de las jornada completa obtienen mejores resultados debido al impacto efectivo de la jornada.

Teniendo en cuenta estos resultados y la importancia de las pruebas Saber 11 para las instituciones educativas públicas y privadas, se puede afirmar que el nivel de educación de las instituciones educativas de carácter oficial está por debajo del nivel de las instituciones educativas no oficiales (o privadas). En relación con esta problemática, se escuchan voces de investigadores que señalan la necesidad de establecer planes de mejoramiento de la enseñanza, en los colegios oficiales, acompañados de capacitaciones con miras a la cualificación y profesionalización del profesorado del sector público.

\section{Conclusiones}

Es muy común escuchar en el ambiente educativo las expresiones calidad de la educación, evaluación interna y externa y rendimiento académico, entre otras, como imperativos. Sin

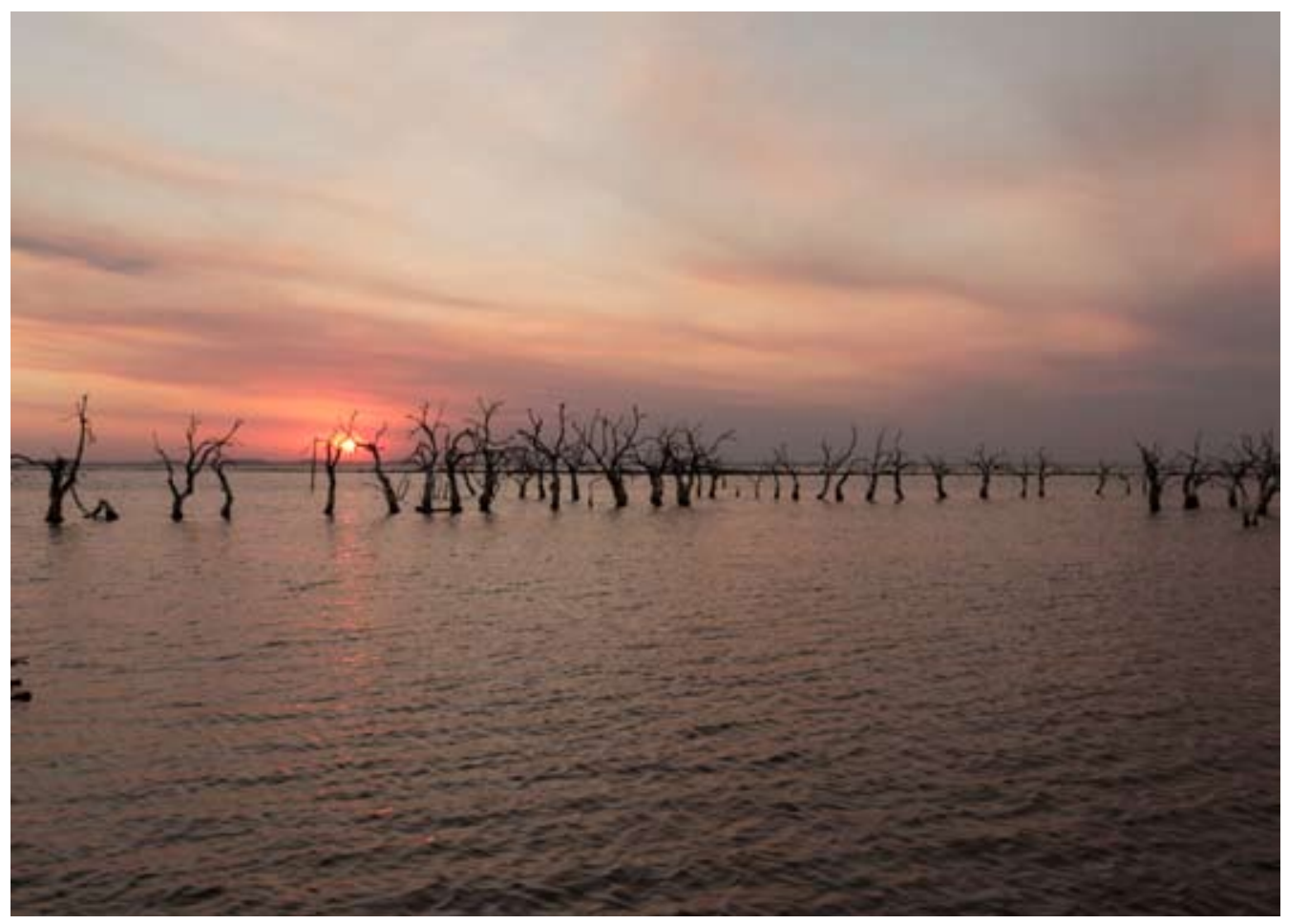

S/T, fotografía. Patricia Bonjour 
embargo, no se tiene en cuenta la realidad social y educativa en que se encuentra la educación pública en el país, la situación laboral de los maestros y la poca preocupación de los distintos gobiernos por financiarla. Sólo se perciben juicios que la desacreditan y que responsabilizan a los docentes de los resultados obtenidos por los estudiantes en sus procesos de enseñanza y de aprendizaje y en las pruebas presentadas.

Los actores centrales de los procesos de enseñanza y de aprendizaje son el estudiante, la escuela y el docente, puesto que el estudiante es un sujeto en constante formación y para ésta son indispensables la escuela y el docente. Pero, también es cierto que, actualmente, en el mundo del mercado, del consumo y de la producción, se asocia la calidad de la educación" con estrategias del poder para convertir la educación en una mercancía. Una evidencia de estos propósitos es la exigencia para que las instituciones, tanto públicas como privadas, se adecuen a modelos educativos extranjeros.

Estas posiciones críticas ven las pruebas Saber 11 como un pretexto para certificar la calidad de la educación pública que, con la promesa de mejorarla, de hacerla competitiva, abre el camino para privatizarla y para que comience a funcionar como una empresa desde la perspectiva neoliberal.

Con el propósito cada día más frecuente, por parte de los entes gubernamentales, de establecer el rendimiento de los estudiantes mediante sistemas estandarizados de evaluación, cuyos resultados son usados para determinar la calidad de la educación, se crea una situación controversial al querer determinar la calidad educativa de los colegios basándose en los resultados de este tipo de pruebas, máxime cuando se equiparan las instituciones que pertenecen a contextos socioeconómicos distintos $y$, por ende, sus condiciones son significativamente diferentes. Además, algunos investigadores consideran que este tipo de evaluación se limita a evidenciar solamente ciertas competencias de los individuos examinados, desconociendo otras y, más grave aún, desconociendo los fines mismos de la educación.

Sin desconocer la validez de estas posturas, igualmente, se presentan otras que estiman necesario reconocer que estas pruebas son una base para diseñar estrategias de calidad educativa y rendimiento académico en las instituciones educativas. Como consecuencia y con base en los resultados de la investigación que se expone en este artículo, se perfilan una serie de acciones para mejorar sus resultados: a) diseñar planes de mejoramiento y estrategias pedagógicas para fortalecer cada una de las áreas analizadas; b) capacitar al cuerpo docente, dignificarlo, reconocer su identidad política y social, su compromiso por la defensa de la educación pública, sus derechos, y reconocer el papel que juega en la sociedad frente a la educación de sus discípulos; c) implementar actividades que le permitan a la comunidad educativa y estudiantil adquirir un mejor nivel de aprendizaje, para la obtención de los logros esperados; d) establecer, desde la Secretaría de Educación, planes de seguimiento y acompañamiento a los docentes con cursos de capacitación y profesionalización tendientes a mejorar los resultados obtenidos, con recursos y financiación de acuerdo con la realidad económica y social de la educación pública del país; e) diseñar, junto con los docentes, los consejos académicos, los estudiantes y la comunidad educativa de los colegios públicos, las diferentes formas de evaluación, tanto interna como externa, acordes a las necesidades de Colombia, incluidas las pruebas Saber 11, y f) instaurar, de manera integral y con todos los colegios públicos, distintas formas de evaluación que analicen otras variables que son parte de la formación y del desarrollo del estudiante, tales como: su estrato social, el contexto geográfico del colegio, su familia, las condiciones locativas de la institución, su financiación y los recursos didácticos, entre otros.

Notas

1 Prueba Saber 11: evaluación enfocada a evidenciar el desarrollo de competencias, se aplica a nivel nacional a los estudiantes de grado 11 de las instituciones públicas y privadas, a través del Instituto Colombiano para el Fomento de la Educación Superior (ICFES), con el propósito de monitorear la calidad de la educación de las instituciones.

2 PISA: Programa Internacional para la Evaluación de Estudiantes (Programme for International Student Assessment)), tiene por objeto evaluar hasta qué punto los alumnos cercanos al final de la educación obligatoria han adquirido algunos de los conocimientos y habilidades necesarios para la participación plena en la sociedad del saber. PISA saca a relucir aquellos países que han alcanzado un buen rendimiento y, al mismo 
tiempo, un reparto equitativo de oportunidades de aprendizaje, ayudando así a establecer metas ambiciosas para otros países. Las pruebas PISA son aplicadas en Colombia cada tres años. Examinan el rendimiento de alumnos de 15 años en áreas temáticas clave y estudian igualmente una gama amplia de resultados educativos, entre los que se encuentran: la motivación de los alumnos por aprender, la concepción que éstos tienen sobre sí mismos y sus estrategias de aprendizaje. Cada una de las tres evaluaciones pasadas de PISA se centró en un área temática concreta: la lectura (en 2000), las matemáticas (en 2003) y las ciencias (en 2006); siendo la resolución de problemas un área temática especial en PISA 2003. El programa está llevando a cabo una segunda fase de evaluaciones en el 2009 (lectura), 2012 (matemáticas) y 2015 (ciencias).

3 SPSSes un programa estadístico informático muy usado en las ciencias exactas, sociales y aplicadas, además de las empresas de investigación de mercado. Originalmente SPSS fue creado como el acrónimo de Statistical Package for the Social Sciences aunque también se ha referido como "Statistical Product and Service Solutions” (Pardo, A., \& Ruiz, M.A., 2002, p. 3)

4 Según la normatividad vigente relativa al calendario académico y al plan de estudios, se brindan cuatro jornadas escolares, entendidas éstas, como el tiempo que la institución educativa dedica a los estudiantes para la prestación del servicio público educativo.

\section{Referencias}

Diario Oficial No. 44.901 jueves 15 de agosto de 2002. DecretoNúmero 1850 DE 2002 (agosto 13)

Evaluación Bogotá (2016) Evaluación Externa. Plan Operativo Anual (POA) Recuperado de:http://evaluacion. educacionbogota.edu.co/index.php/evaluacion-delos-aprendizajes/pruebas-externas

Gómez, C. (2016) «Clasificación de colegios según las Pruebas Saber 11 del ICFES: un análisis usando modelos marginales (MM)». Sociedad y economía, No. 30 (enero - junio 2016): 69-89.

ICFES (2003) Programa de evaluación de la educación básica Pruebas Saber lenguaje y matemáticas grados 3, 5, 7 y 9 . Subdirección de Aseguramiento de la Calidad. Colombia. Recuperadode:https:/www.researchgate. net/profile/Yuly_Vanegas/publication/282442796_ Fundamentacion_conceptual_Pruebas_SABER_ICFES/links/5610331a08aec422d115017e. pdf?origin=publication_list

ICFES (2010). Sistema Nacional de Información de Evaluación Educativa SNIEE. p. 10.

Mejía, M. R. (2000) La calidad de la educación en tiempos de globalización. Una mirada crítica desde la educación popular. Ponencia presentada al Congreso Pedagógico Nacional de Fe y Alegría. Nicaragua, 2122 de octubre de 2000.

Ministerio de Educación (2010) Educación de Calidad el Camino para la Prosperidad. Recuperado de: http://www.mineducación.gov.co/cvn/1665/articles237397 Archivo pdf.pdf

Ministerio de Educación Nacional (S.F.) Plan Decenal de Educación 1996-2005. Recuperado de: http:// www.plandecenal.edu.co/cms/media/herramientas/ pnde_1996_2005.pdf

Ministerio de Educación Nacional (2013) Sistema de evaluación. Revista Altablero No. 24. Sep-Oct. Recuperado de: http://www.mineducacion.gov.co/1621/ propertyvalue-31335.html

Revista Semana.com (2014) ¿Qué hace que un estudiante tenga buenos resultados académicos? Recuperado de: http://www.semana.com/educacion/articulo/quehace-que-un-estudiante-tenga-buenos-resultadosacademicos/408203-3

Secretaría de Educación del Distrito (S.F.) Subsecretaría de Calidad y Pertinencia Dirección de Evaluación de la Educación. Seice Sistema de evaluación integral para la calidad de la educación.Alcaldía Mayor de Bogotá.

\section{Bibliografía}

Bonilla, L. (2011) Doble jornada escolar y calidad educativa. Documentos de Trabajo sobre Economía Regional. Nro. 143.

Canavos, George C. (1998) Probabilidad y Estadística. Aplicaciones y Métodos. México: McGraw-Hill.

Magendzo, A. (1988) Preguntas y respuestas desafiantes en torno a la calidad de la educación. PIIE. Santiago de Chile.

Pardo, A., \& Ruiz, M.A. (2002) SPSS 11. Guia para el análisis de datos. $1^{\mathrm{a}}$ ed., Madrid: McGraw-Hill Interamericana de España S.L.

Fecha de Recepción: 17 de mayo de 2017

Primera Evaluación: 12 de junio de 2017

Segunda Evaluación: 25 de junio de 2017

Fecha de Aceptación: 22 de agosto de 2017 